\title{
Polyhedral meshing as an innovative approach to computational domain discretization of a cyclone in a fluidized bed CLC unit
}

\author{
Marcin Sosnowski ${ }^{1, *}$, Jaroslaw Krzywanski ${ }^{1}$, and Renata Gnatowska ${ }^{2}$ \\ ${ }^{1}$ Jan Dlugosz University in Czestochowa, Faculty of Mathematics and Natural Sciences, 13/15 Armii \\ Krajowej Av, 42-200 Czestochowa, Poland \\ ${ }^{2}$ Czestochowa University of Technology, Faculty of Mechanical Engineering and Computer Science, \\ 21 Armii Krajowej Av, 42-200 Czestochowa, Poland
}

\begin{abstract}
Chemical Looping Combustion (CLC) is a technology that allows the separation of $\mathrm{CO}_{2}$, which is generated by the combustion of fossil fuels. The majority of process designs currently under investigation are systems of coupled fluidized beds. Advances in the development of power generation system using CLC cannot be introduced without using numerical modelling as a research tool. The primary and critical activity in numerical modelling is the computational domain discretization. It influences the numerical diffusion as well as convergence of the model and therefore the overall accuracy of the obtained results. Hence an innovative approach of computational domain discretization using polyhedral (POLY) mesh is proposed in the paper. This method reduces both the numerical diffusion of the mesh as well as the time cost of preparing the model for subsequent calculation. The major advantage of POLY mesh is that each individual cell has many neighbours, so gradients can be much better approximated in comparison to commonly-used tetrahedral (TET) mesh. POLYs are also less sensitive to stretching than TETs which results in better numerical stability of the model. Therefore detailed comparison of numerical modelling results concerning subsection of CLC system using tetrahedral and polyhedral mesh is covered in the paper.
\end{abstract}

\section{Introduction}

\subsection{Simulation of fluidized bed CLC unit}

The combustion of fossil fuels in energy production and transportation is the main source of climate change. Therefore the development of new combustion technologies characterized by low pollutant emission rates is in the spotlight of contemporary research. Therefore the Circulating Fluidized Bed (CFB) boilers are popular alternatives to the traditional pulverized coal boilers mostly due to decreased emission, low combustion temperature and

\footnotetext{
* Corresponding author: m.sosnowski@ajd.czest.pl
} 
high fuel flexibility. In order to segregate the $\mathrm{CO}_{2}$, the Chemical Looping Combustion (CLC) was introduced to CFB designs. The principle of CLC was developed and patented in 1954 by Lewis and Gilliland [1]. A key factor in the CLC performance is the Oxygen Carrier (OC), which circulates between the air reactor and fuel reactor (Fig. 1). Apart from the $\mathrm{OC}$ also the process design is important. Generally it consists of two reactors: the air reactor in which the OC is loaded with oxygen and the fuel reactor where the OC supplies the oxygen for fuel combustion. The majority of process designs currently under investigation are systems of coupled fluidized beds. The system analysed within the confines of the research is based on a design presented in [2] with bubbling fluidized bed as fuel reactor and a fast fluidized bed riser as air reactor.

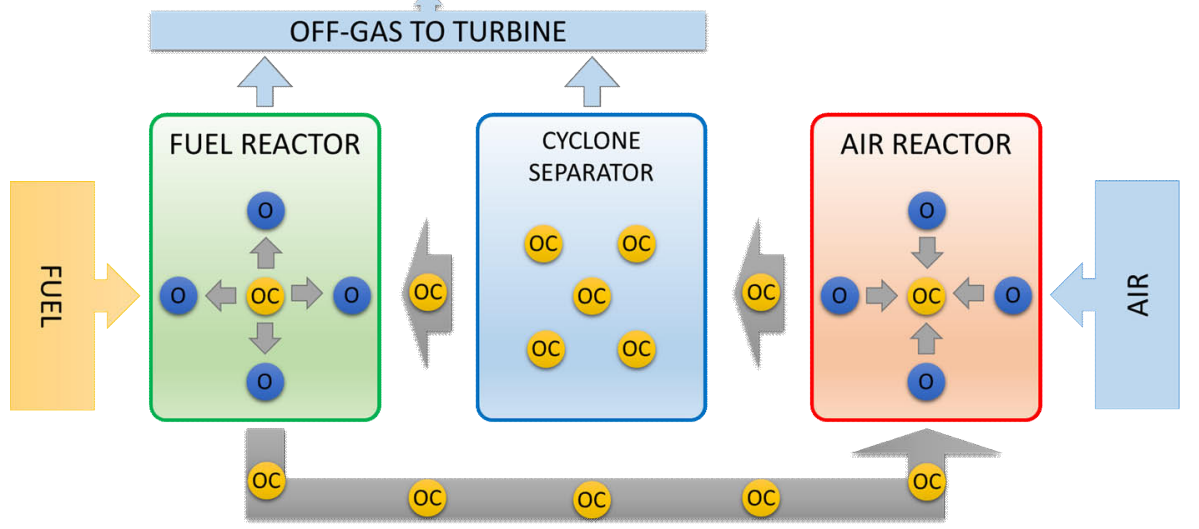

Fig. 1. Schematic diagram of CLC (O - oxygen, OC - oxygen carrier).

The heat and mass transfer between the phases, the chemical reactions and complex hydrodynamics of CFBs are still not completely understood and difficult to predict despite its widespread applications. The knowledge concerning CFB systems relied mostly on experimental studies but in recent years researchers have focused on improving CFB efficiency by utilizing the capabilities of numerical methods. With the rapid development of high performance computers, various techniques such as artificial intelligence including neurocomputing and fuzzy logic approach [3-7] were used in CFB modelling [8, 9] but Computational Fluid Dynamics (CFD) turned out to be one of the best tools in such research and plays an important role in the design and optimization of industrial systems [10]. A common challenge for CFD is the expensive computational cost in terms of time required to reach a converged solution. The very first issue influencing the CPU-time is the computational domain discretization, therefore the research carried out within the confines of this paper focus on the innovative approach to discretization of a fluidized bed CLC unit.

\subsection{Polyhedral meshing}

Advances in aerospace [11], automotive [12], civil engineering [13], medicine [14] and other numerous branches of industry could not have been achieved without applying numerical modelling as a research tool. The primary and critical activity in numerical modelling is the computational domain discretization. This process consists in dividing the analysed geometry into numerous small control volumes and it is commonly called "meshing". In fluid dynamics applications it is the most desirable to divide the domain into hexahedral (HEX) control volumes denoted red in Fig. 2 as the resulting mesh is characterized by low numerical diffusion particularly in case of flow perpendicular to the faces of control volumes. Unfortunately it is not always possible to construct structured HEX mesh for complex geometries or it can be particularly time-consuming part of CFD 
analysis. Therefore semi-automatic tetrahedral (TET) mesh generation algorithms have been implemented into commercial pre-processors. TETs denoted blue in Fig. 2 are the simplest volume elements as they are built out of four faces. The faces are plane segments, so both face and volume centroid locations are well defined. The unquestionable advantage of TET mesh is the ease of its generation even in case of complicated geometry. On the other hand TETs cannot be stretched excessively, so significantly larger number of elements has to be used in comparison to structured HEX mesh in order to achieve a reasonable accuracy in boundary layers, long channels or small gaps. Moreover TETs have only four neighbours, therefore computing gradients can be problematic due to spatial position of neighbour nodes, which may lie in nearly one plane. In consequence, the numerical diffusion of TET mesh is significantly higher than HEX mesh and low quality TETs result in convergence errors and significantly reduce the accuracy of the solution.

The polyhedral (POLY) mesh denoted green in Fig. 2 was introduced in STAR-CCM+ solver and ANSYS Fluent in order to combine the advantages of HEX (low numerical diffusion resulting in accurate solution) and TET mesh (rapid semi-automatic generation) as well as to overcome the disadvantages of both the above mentioned types of control volumes.

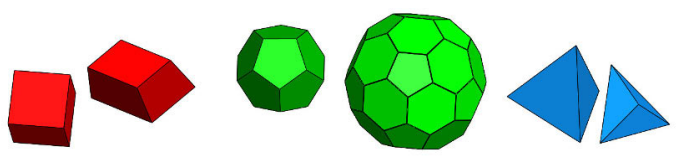

Fig. 2. Hexahedral (red), polyhedral (green) and tetrahedral (blue) control volumes.

The major benefit of POLYs is that each individual cell has many neighbours, so gradients can be much better approximated in comparison to TETs. POLYs are also less sensitive to stretching than TETs which results in better numerical stability of the model. In some cases, POLYs can even achieve better accuracy than HEXs due to larger number of neighbouring elements. They allow the exchange of mass over a larger number of faces, reducing numerical diffusion effects caused by flows not perpendicular to any of the cell faces. This proves to be advantageous in situations where no prevailing flow direction can be identified and leads to a more accurate solution achieved with a lower cell count.

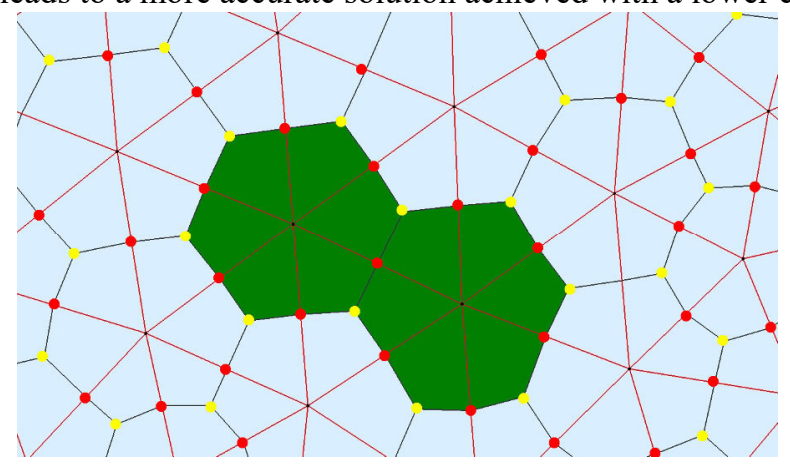

Fig. 3. TET to POLY conversion: edge centroids (red dots) and face centroids (yellow dots) of TETs.

POLY mesh generation in commercial CFD code like ANSYS Fluent consists in simple conversion of TET elements to POLYs by decomposition of cell into multiple sub-volumes depicted in Fig. 3. In order to do so, new edges are created on each face between the face centroid (yellow dots in Fig. 3) and the centroids of the edges of that face (red dots in Fig. 3). Subsequently new faces are created within the cell by connecting the cell centroid to the new edges on each face. The newly-created faces may be adjusted and merged with neighbouring faces during the agglomeration process in order to minimize the number of faces on the resultant polyhedral cell. 


\section{Methods}

\subsection{Research object}

The lab-scale fluidized bed CLC unit was the object of the carried out research. The unit was designed on the basis of the design presented by Thon et al. [2] and it was divided into three main parts: fuel reactor, air reactor and cyclone separator (Fig. 4). The last mentioned is the most demanding part of the unit in terms of computational fluid dynamics therefore it was selected for detailed analysis in the preliminary stage of the research concerning the evaluation of polyhedral mesh application in discretization of the computational domain.
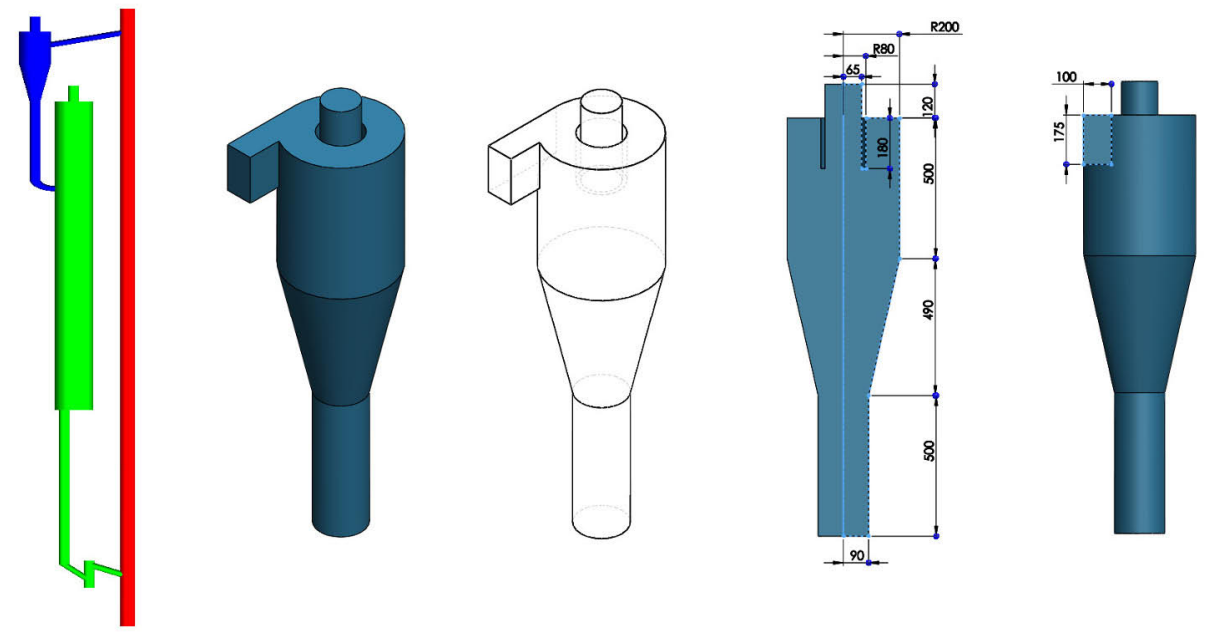

Fig. 4. The analysed fluidized bed CLC unit: air reactor (red), fuel reactor (green), cyclone separator (blue) and detailed view of the cyclone.

\subsection{Analysed configurations of computational domain discretization}

The computational domain was discretized with TET cells (Fig. 5) using ANSYS Meshing. Six configurations of input parameters were used in order to generate TET meshes of number of cells ranging from approx. $35 \mathrm{k}$ to $516 \mathrm{k}$.

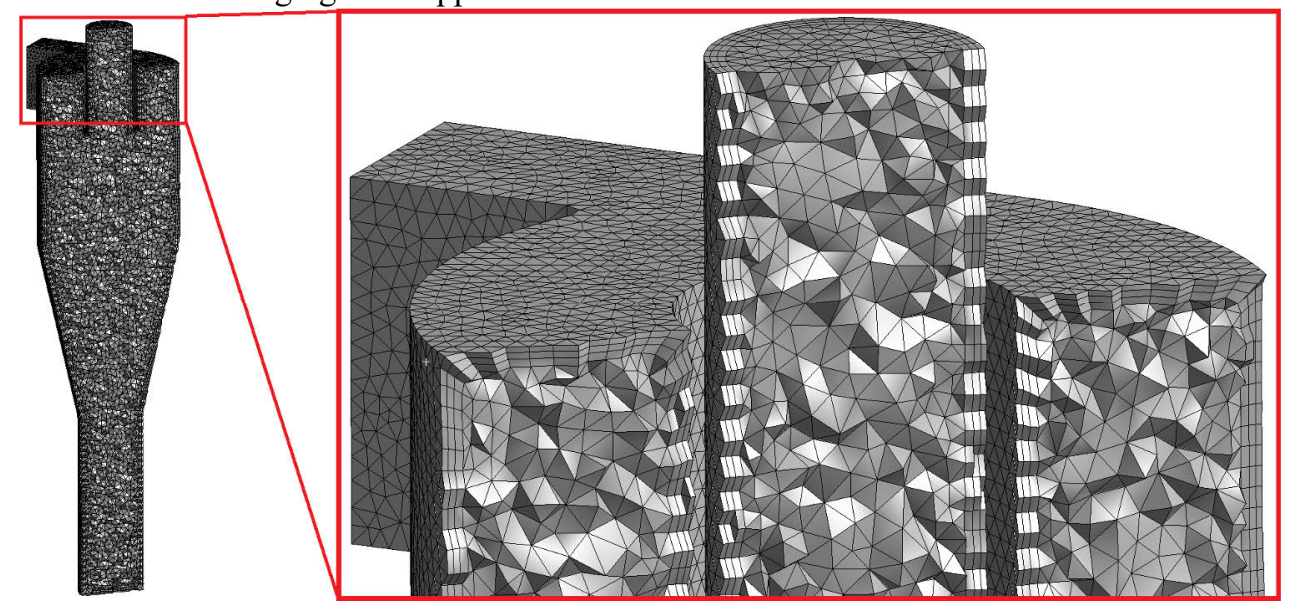

Fig. 5. The computational domain discretization with TET mesh. 
The uniform size function with specified max face size and max TET size according to Fig. 6 was used. Moreover inflation of 3 layers, growth rate equal 1.2 and maximum thickness as specified in Fig. 6 was generated by post algorithm on all surfaces except for inlet and outlet. Each TET mesh was converted to POLY mesh in ANSYS Fluent.

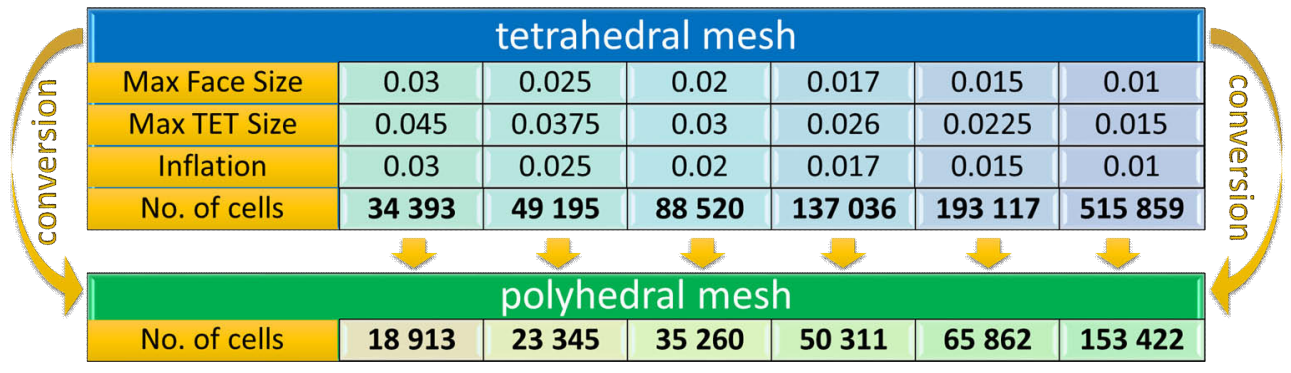

Fig. 6. Parameters of investigated meshes.

The minimum orthogonal quality for both the TET mesh and POLY mesh did not decrease below 0.11 (orthogonal quality ranges from 0 to 1 , where values close to 0 correspond to low quality) and maximum ortho skew did not exceed 0.89 (ortho skew ranges from 0 to 1 , where values close to 1 correspond to low quality). These quality factors indicate good quality of the generated meshes.

\subsection{Reference mesh}

The structured HEX reference mesh of approx. one million cells was generated in order to validate the results obtained using TET and POLY meshes of various number of cells. The HEX elements are considered as the most desirable in CFD analysis and the mesh-dependency studies carried out within the research proved, that the solution obtained with approx. one million cells is mesh-independent. Therefore the results obtained by calculations using the reference mesh are considered as the baseline for subsequent analysis of evaluation of POLY mesh application in discretization of the computational domain.

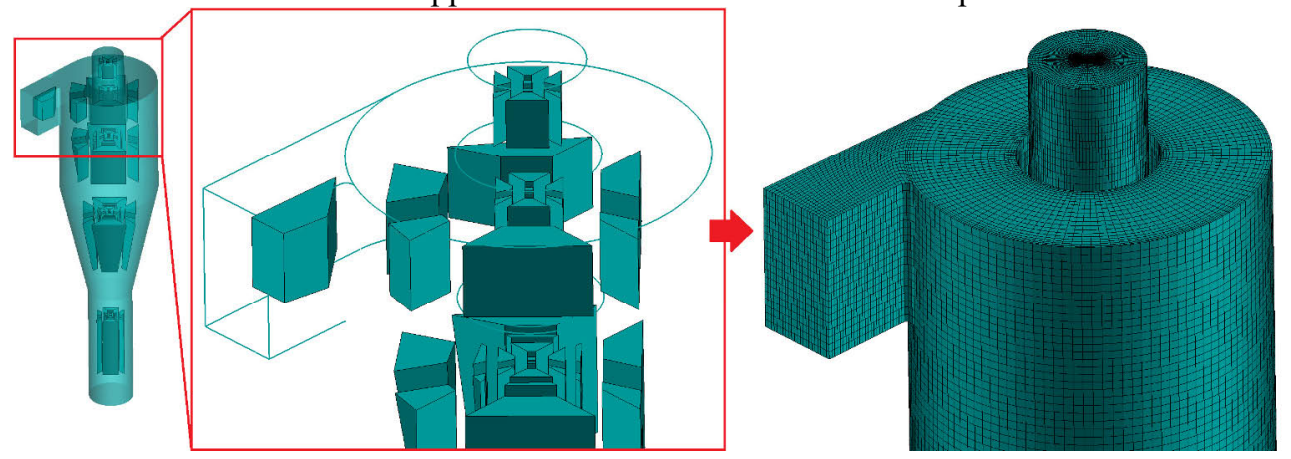

Fig. 7. Reference mesh: blocking and output mesh.

The structured HEX mesh was constructed in ANSYS ICEM CFD by applying the blocking technique. The final blocking topology consisted of 54 blocks created by splitting the initial block in order to describe the main part of the cyclone separator plus one additional block created by extruding the face representing the inlet duct. Triple o-grid was applied to arrange mesh lines into an "O" shape to reduce skew in cases of a block corner located on a continuous curve as depicted in Fig. 7. 


\subsection{Computational model}

The exactly identical computational model (except for the mesh) was used for all thirteen cases (reference HEX mesh, six configurations of TET mesh and six configurations of POLY mesh obtained by conversion of TET mesh in ANSYS Fluent) analysed within the research. The solver was configured as pressure-based and the analysis were performed for steady state. Standard k-epsilon viscous model was used along with standard wall functions. The viscous model constants are listed in Table 1.

Table 1. k-epsilon viscous model constants.

\begin{tabular}{|c|c|}
\hline Model Constant & Value \\
\hline Cmu & 0.09 \\
\hline C1-Epsilon & 1.44 \\
\hline C2-Epsilon & 1.92 \\
\hline TKE Prandtl Number & 1 \\
\hline TDR Prandtl Number & 1.3 \\
\hline
\end{tabular}

Velocity-inlet boundary condition type was assigned to the inlet (green surface in Fig. 8 ) with velocity magnitude normal to the boundary equal $5 \mathrm{~m} / \mathrm{s}$. The turbulence parameters were specified as intensity and hydraulic diameter equal $5 \%$ and $0.127 \mathrm{~m}$ respectively.
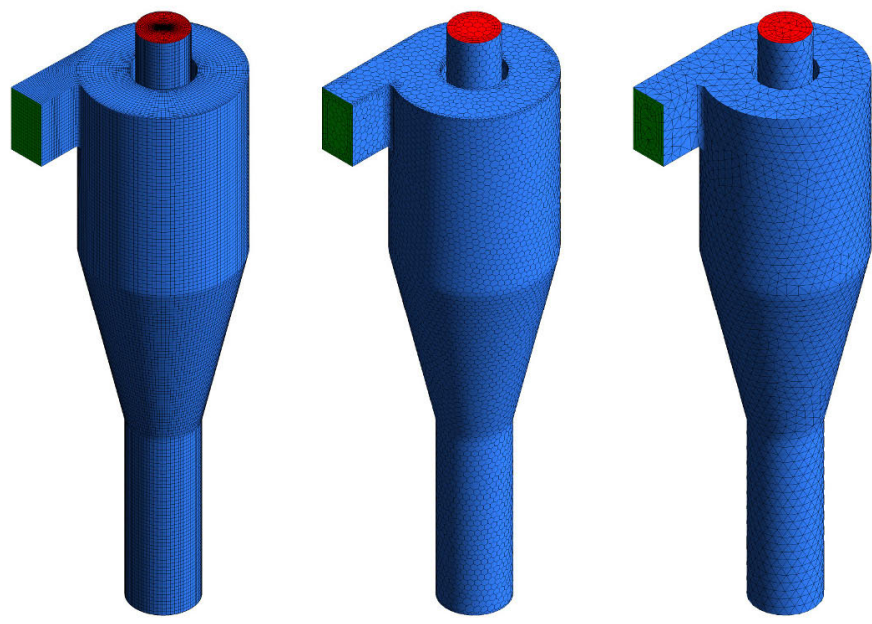

Fig. 8. Boundary conditions assigned to HEX, POLY and TET mesh: velocity-inlet (green), pressure-outlet (red), wall (blue).

The outlet of the computational domain (red surface in Fig. 8) was defined as pressure-outlet with gauge pressure of $0 \mathrm{~Pa}$ (operating pressure $-101325 \mathrm{~Pa}$ ), backflow turbulent intensity of $5 \%$ and backflow hydraulic diameter of $0.15 \mathrm{~m}$.

Wall type condition was assigned to the remaining surfaces (blue surfaces in Fig. 8). The roughness height was set to $0 \mathrm{~m}$ and roughness constant to 0.5 .

The solution was assumed as converged when residuals of $\mathrm{x}$-velocity, y-velocity, z-velocity, k, epsilon decreased below 1e-5 and continuity residual decreased below 1e-3. Moreover three additional surface monitors were defined as integral of velocity magnitudes on outlet surface, XY plane and YZ plane.

Pressure-velocity coupling by SIMPLE algorithm was used as a solution method. This algorithm uses a relationship between velocity and pressure corrections to enforce mass conservation and to obtain the pressure field. 


\section{Results}

The results showed in Fig. 9 are presented as velocity contour plots on XY and YZ planes for the reference HEX mesh, POLY meshes and TET meshes for all configurations. It allows detailed qualitative and rough quantitative comparison of the results in respect of mesh type and number of elements.

Integrated velocities on the outlet surface and XY plane were normalized to the values obtained for the reference HEX mesh and are shown in Fig. 10. Each one of the six cases is presented for the reference HEX mesh, TET mesh and POLY mesh obtained by conversion of the respective TET mesh in ANSYS Fluent according to the data listed in Fig. 6.
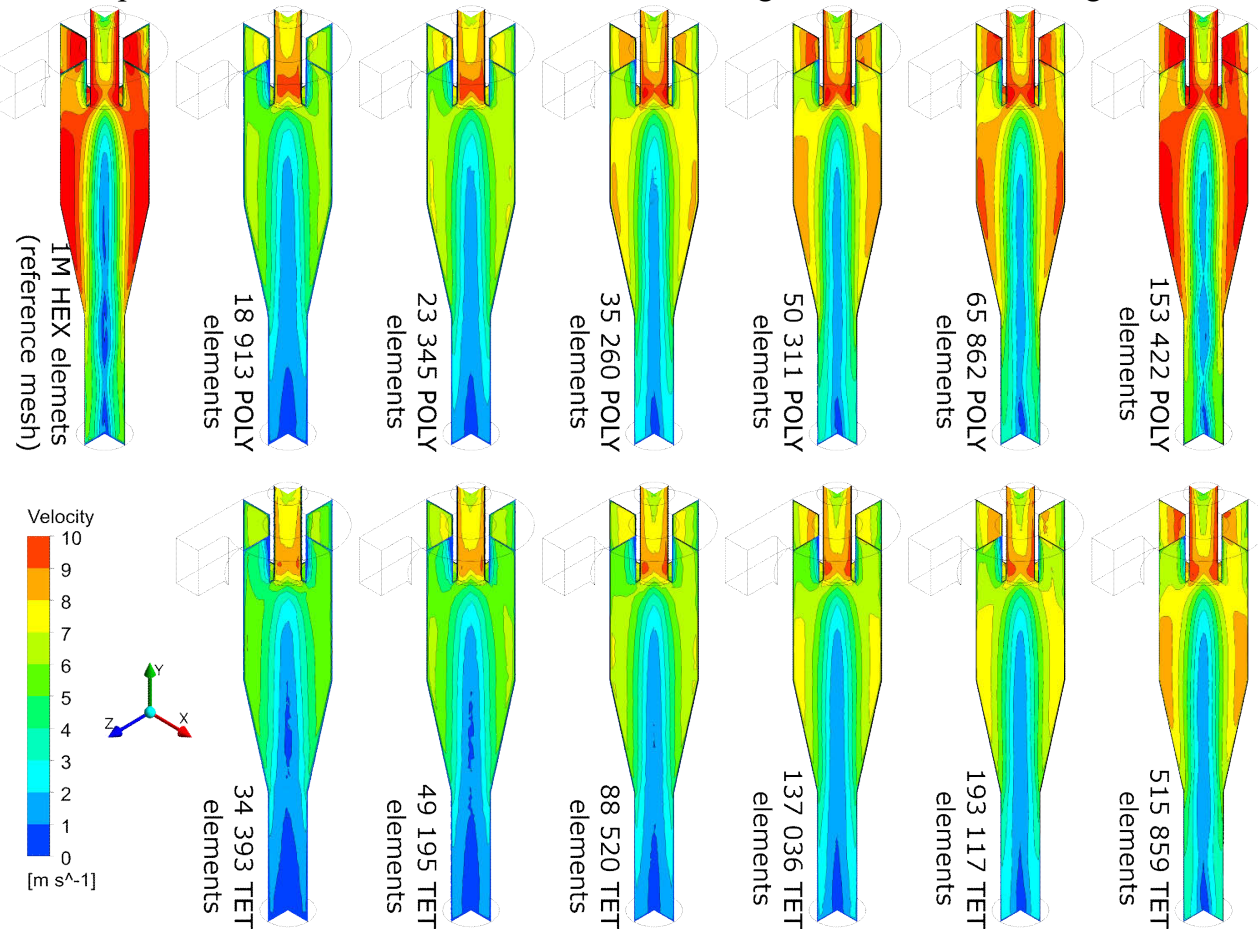

Fig. 9. Velocity on $\mathrm{XY}$ and $\mathrm{YZ}$ plane for all analysed meshes.
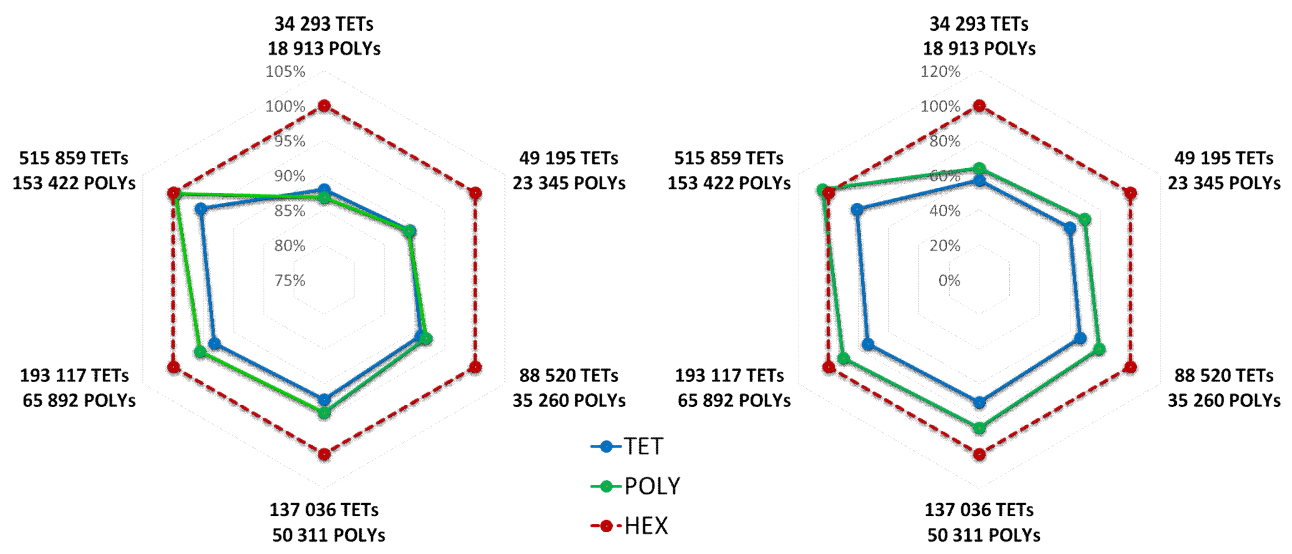

Fig. 10. Integrated velocity on outlet surface (left) and XY plane (right) for analysed POLY and TET meshes normalized to reference HEX mesh. 

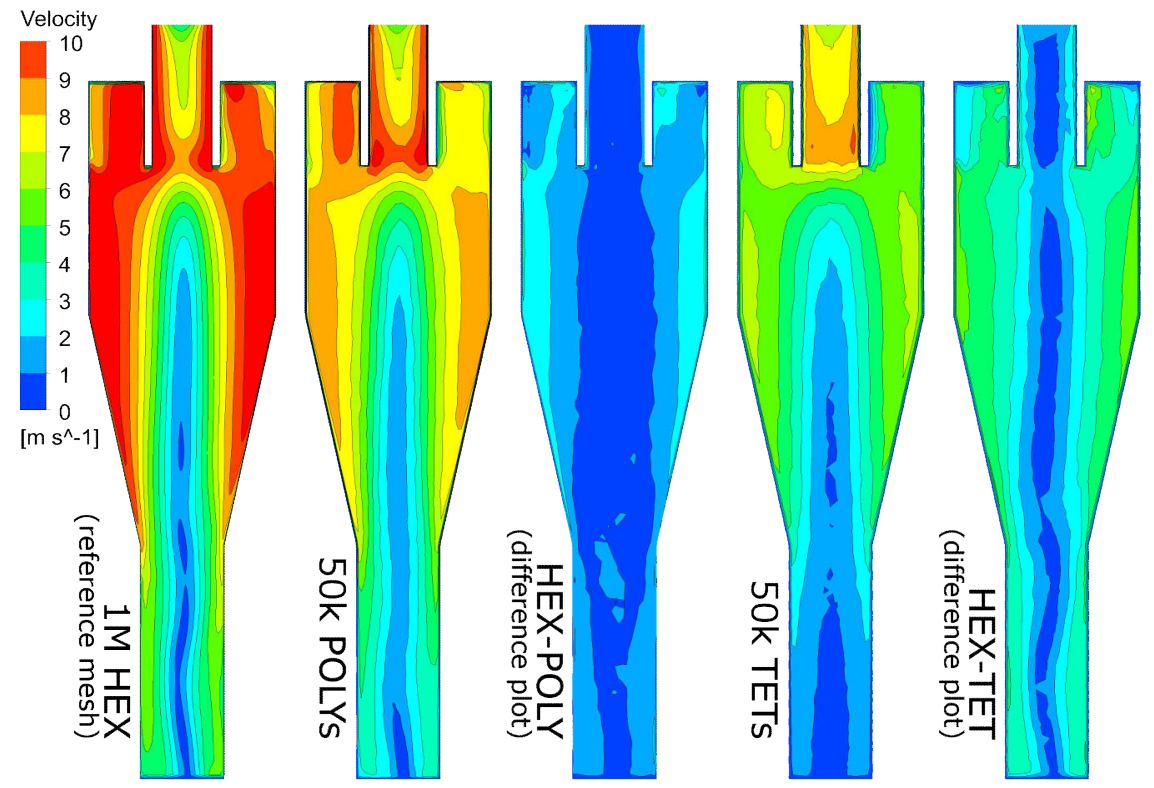

Fig. 11. Velocity on XY plane for analysed meshes with difference plots of HEX-POLY and HEX-TET.

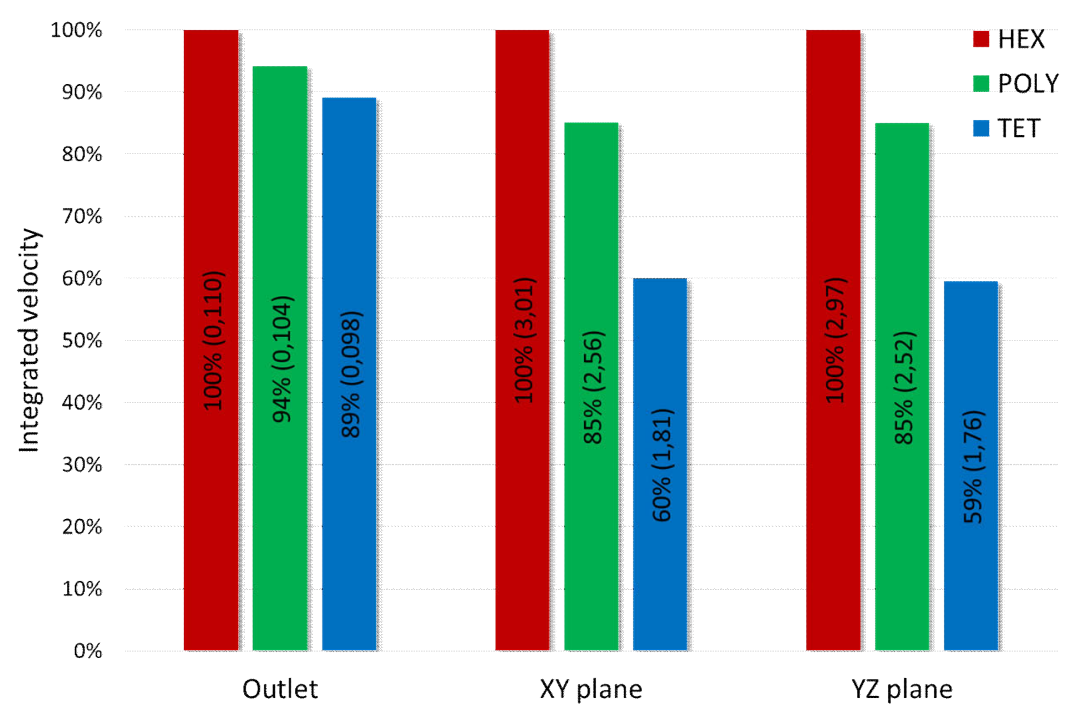

Fig. 12. Integrated velocity on outlet surface, $X Y$ plane and $Y Z$ plane for POLY, TET and the reference HEX mesh.

Fig. 11 is presented in order to compare the results obtained using TET and POLY meshes of comparable number of elements (50 311 POLYs and 49195 TETs). The figure shows the velocity in XY plane for the reference HEX mesh, POLY and TET meshes as well as difference plots generated by subtracting the local velocity for reference mesh and POLY or TET mesh respectively.

The quantitative outlook for the solution accuracy is presented in Fig. 12, which shows comparison of the integrated velocity on outlet surface, $\mathrm{XY}$ and $\mathrm{YZ}$ planes obtained with 
the analysed mesh types. The integrated velocity is shown as absolute values as well as normalized to the values generated with the reference HEX mesh.

Despite the results accuracy, the calculation time is also a very important factor in terms of the overall cost of the analysis. The POLY mesh turned out to be beneficial in this field as the overall calculation time in case of POLY mesh is approx. three to five times shorter in comparison to the respective TET mesh depending on the analysed cases.

\section{Discussion}

The aim of the carried out research was to investigate the possibility of applying POLY meshing as an innovative approach to computational domain discretization of a fluidized bed CLC unit. Six TET meshes of number of elements ranging from approx. $34 \mathrm{k}$ to $516 \mathrm{k}$ were prepared as well as six POLY meshes obtained by conversion of the respective TET meshes in ANSYS Fluent. Reference HEX mesh of approx. 1M elements was used as a baseline and results obtained for all TET and POLY meshes were referenced to it.

As can be concluded on the basis of Fig. 9 and Fig. 10, the results obtained with POLY mesh are significantly more accurate than TET mesh even though the element count for POLY meshes was from 1.8 to 3.4 times lower. The velocity distribution obtained for the TET mesh of 516k elements is comparable to POLY mesh of 50k elements.

The significant difference between POLY and TET meshes of comparable number of elements (approx. 50k) can be determined on the basis of Fig. 11. The velocity contour plot for TET is not even comparable to the reference HEX mesh, while POLY is in a good qualitative agreement and quantitative difference of approx. $10 \%-15 \%$. This thesis is proved by Fig. 12, where the integrated velocity on XY plane for TET mesh is only $60 \%$ of the reference value while in the case of POLY mesh it is $85 \%$. It has to be mentioned that discretizing the cyclone separator domain by $50 \mathrm{k}$ elements generates rather coarse mesh and $85 \%$ agreement obtained for POLY mesh is a very good result.

Moreover the POLY mesh of $153 \mathrm{k}$ elements indicates $100 \%$ agreement with the reference mesh in terms of integrated velocity on outlet surface and almost $100 \%$ agreement in terms of integrated velocity on XY plane. These factors for almost three times finer TET mesh (516k elements) are respectively in $95 \%$ and $81 \%$ agreement.

Therefore the overall conclusion from the carried out research is that the major benefit of POLY mesh consists in the more accurate solution obtained with fewer cells, which in consequence significantly reduces the computational time.

The carried out research revealed a great potential in discretization of computational domain using POLY mesh but further investigation is necessary in order to validate this innovative technique with transient multiphase flow, advanced turbulence models and different oxygen carriers. Moreover the influence of the under-relaxation factors as well as different algorithms of pressure-velocity coupling on the convergence time has to be analysed.

\section{References}

1. Gilliard, E. and W. Lewis, Production of pure carbon dioxide. 1954.

2. Thon, A., et al., Operational experience with a system of coupled fluidized beds for chemical looping combustion of solid fuels using ilmenite as oxygen carrier. Applied Energy, 2014. 118: p. 309-317.

3. Krzywanski, J. and W. Nowak, Modeling of bed-to-wall heat transfer coefficient in a large-scale CFBC by fuzzy logic approach. International Journal of Heat and Mass Transfer, 2016. 94: p. 327-334. 
4. Krzywanski, J., et al., A generalized model of SO2 emissions from large- and smallscale CFB boilers by artificial neural network approach Part 1. The mathematical model of SO2 emissions in air-firing, oxygen-enriched and oxycombustion CFB conditions. Fuel Processing Technology, 2015. 137: p. 66-74.

5. Krzywanski, J., et al., A generalized model of SO2 emissions from large- and smallscale CFB boilers by artificial neural network approach Part 2. SO2 emissions from large- and pilot-scale $C F B$ boilers in $O-2 / N-2, O-2 / C O 2$ and $O-2 / R F G$ combustion atmospheres. Fuel Processing Technology, 2015. 139: p. 73-85.

6. Krzywanski, J. and W. Nowak, Artificial Intelligence Treatment of SO2 Emissions from CFBC in Air and Oxygen-Enriched Conditions. Journal of Energy Engineering, 2016. 142(1).

7. Krzywanski J., et al., The Non-Iterative Estimation of Bed-to-Wall Heat Transfer Coefficient in a CFBC by Fuzzy Logic Methods. Procedia Engineering, 2016, 157, p. 66-71.

8. Krzywanski, J., R. Rajczyk, and W. Nowak, Model research of gas emissions from lignite and biomass co-combustion in a large scale CFB boiler. Chemical and Process Engineering-Inzynieria Chemiczna I Procesowa, 2014. 35(2): p. 217-231.

9. Krzywanski, J., et al., A 1.5D model of a complex geometry laboratory scale fuidized bed CLC. Equipment. Powder Technology, 2016.

10. Li, T.W., et al., CFD simulations of circulating fluidized bed risers, part I: Grid study. Powder Technology, 2014. 254: p. 170-180.

11. Kroll, N., The ADIGMA Project, in Adigma-a European Initiative on the Development of Adaptive Higher-Order Variational Methods for Aerospace Applications: Results of a Collaborative Research Project Funded by the European Union, 2006-2009, N. Kroll, et al., Editors. 2010, Springer-Verlag Berlin: Berlin. p. 1-9.

12. Jamrozik, A., et al., Numerical simulation of two-stage combustion in SI engine with prechamber. Applied Mathematical Modelling, 2013. 37(5): p. 2961-2982.

13. Gnatowska, R., A Study of Downwash Effects on Flow and Dispersion Processes around Buildings in Tandem Arrangement. Polish Journal of Environmental Studies, 2015. 24(4): p. 1571-1577.

14. Spiegel, M., et al., Tetrahedral vs. polyhedral mesh size evaluation on flow velocity and wall shear stress for cerebral hemodynamic simulation. Computer Methods in Biomechanics and Biomedical Engineering, 2011. 14(1): p. 9-22. 